\title{
Factores pronóstico asociados a parto prematuro en mujeres a quienes se les practicó apendicectomía durante el embarazo en un país de medianos ingresos
}

\author{
Prognosis factors associated with premature delivery in women undergoing \\ appendectomy during pregnancy in a middle-income country
}

\author{
Kenndy Arévalo¹, Giancarlo Buitrago², Juan Sebastián Moyano33, Rubén Caycedo ${ }^{4}$
}

Médica, residente de Cirugía General, Departamento de Cirugía, Facultad de Medicina, Universidad Nacional de Colombia, Bogotá,
D.C., Colombia
Director, Instituto de Investigaciones Clínicas, Universidad Nacional de Colombia-Hospital Universitario Nacional de Colombia,
Bogotá, D.C., Colombia
Investigador, Instituto de Investigaciones Clínicas, Universidad Nacional de Colombia-Hospital Universitario Nacional de Colombia,
Bogotá, D.C., Colombia
4 Jefe, Departamento de Cirugía, Facultad de Medicina, Universidad Nacional de Colombia-Hospital Universitario Nacional de
Colombia, Bogotá, D.C., Colombia
Primer puesto, Concurso Nacional del Residente Quirúrgico, $45^{\circ}$ Congreso Semana Quirúrgica Nacional, agosto de 2019, Bogotá,
D.C., Colombia

\section{Resumen}

Introducción. El objetivo del estudio fue estimar los factores pronóstico asociados con el parto prematuro y otros resultados clínicos en mujeres embarazadas sometidas a apendicectomía en Colombia.

Métodos. Se llevó a cabo un estudio retrospectivo de cohorte a partir de las bases de datos administrativos, que incluyó mujeres embarazadas afiliadas al sistema de salud contributivo en Colombia y sometidas a apendicectomía, entre enero de 2013 y noviembre de 2016. Se estimaron la tasa de parto prematuro, la tasa de mortalidad a los 30 días, el ingreso materno a la unidad de cuidados intensivos a 30 días, el reingreso de la madre a los 30 días y el bajo peso al nacer. Se utilizaron regresiones logísticas multivariadas para identificar estos tres factores pronóstico.

Resultados. Se incluyeron I.589 mujeres en el estudio. La edad media fue de $26,43 \pm 5,79$ años, el I7,94 \% de las apendicectomías se practicaron en el tercer trimestre, el 6,10 \% fueron apendicectomías laparoscópicas y el 22,03 \% requirió drenaje por peritonitis. Las tasas de parto prematuro, mortalidad a 30 días, ingreso a la

Fecha de recibido: 30/06/2019 - Fecha aceptación: 21/08/2019

Correspondencia: Kenndy Arévalo, Universidad Nacional de Colombia, Facultad de Medicina, Edificio 471, oficina 107, Bogotá, D.C., Colombia. Teléfono: (571) 316-5000, extensión 15031

Correo electrónico: karevalop@unal.edu.co

Citar como: Arévalo K, Buitrago G, Moyano JS, Caycedo R. Factores pronóstico asociados a parto prematuro en mujeres a quienes se les practicó apendicectomía durante el embarazo en un país de medianos ingresos. Rev Colomb Cir. 2020;35:22-31.

https://doi.org/10.30944/20117582.579.

Este es un artículo de acceso abierto bajo una Licencia Creative Commons - BY-NC-ND https://creativecommons.org/licenses/by-ncnd/4.0/deed.es 
unidad de cuidados intensivos a 30 días, reingreso a los 30 días y bajo peso al nacer, fueron $\mathrm{I2}, 08 \%, 0,13 \%, 9,75 \%$, I6,93 \% y 3,34 \%, respectivamente. La edad menor de I8 años, la apendicectomía en el tercer trimestre y el drenaje por peritonitis se asociaron con un mayor riesgo de parto prematuro. El índice de comorbilidad de Charlson, la apendicectomía en el tercer trimestre y el drenaje por peritonitis, se asociaron con un mayor ingreso materno a la unidad de cuidados intensivos.

Conclusiones. La apendicectomía en el tercer trimestre y la apendicitis complicada, son factores pronóstico asociados a parto prematuro en mujeres colombianas embarazadas sometidas a apendicectomía.

Palabras clave: embarazo; tercer trimestre del embarazo; trabajo de parto prematuro; apendicitis; apendicectomía; factores de riesgo.

\section{Abstract}

Introduction: The objective of the study was to estimate the prognostic factors associated with premature delivery and other clinical outcomes in pregnant women undergoing appendectomy in Colombia.

Methods: A retrospective cohort study was conducted from the administrative healthcare records, which included pregnant women affiliated with the contributory health system in Colombia and undergoing appendectomy, between January 2013 and November 2016. The birth rate was estimated, premature delivery rate, the 30-day mortality rate, 30-day maternal admission to Intensive Care Unit (30-ICU), 30-day readmission (30-R-Adm), and low birth weight (LBW). Multivariate logistic regressions were used to identify these three prognostic factors.

Results: 1589 women were included in the study. Mean age was $26.43 \pm 5.79,17.94 \%$ of the appendectomies were performed in the third trimester, 6.10\% were laparoscopic appendectomies and $22 \%$ required peritonitis drainage. The Rates of premature delivery, 30-day mortality, 30-ICU, 30-R-Adm and LBW were I2\%, 0.13\%, 9.75\%, I6.93\% and $3.34 \%$, respectively. Age under I8 years, appendectomy in the third trimester and peritonitis drainage were associated with an increased risk of premature delivery. Comorbidity Charlson Index, appendectomy in the third trimester and peritonitis drainage were associated with an increased maternal admission to ICU.

Conclusions: Appendectomy in the third trimester and complicated appendicitis are prognostic factors associated with premature delivery in Colombian pregnant women undergoing to appendectomy.

Keywords: pregnancy; pregnancy trimester, third; obstetric labor, premature; appendicitis; appendectomy; risk factors .

\section{Introducción}

El abdomen agudo de causa no obstétrica tiene una incidencia de I en cada 500 a 635 mujeres embarazadas ${ }^{1,2}$. La causa más frecuente de abdomen agudo en el embarazo es la apendicitis aguda, con una frecuencia estimada de I de cada 500 a 2.000 embarazadas, seguida de la colecistitis aguda que ocurre en I de cada I.600 a I0.000 embarazos 3,4 .

En el embarazo, la cirugía de causa no obstétrica aumenta el riesgo de resultados desfavorables, principalmente en el feto, que incluye pérdida fetal, trabajo de parto prematuro, parto prematuro y muerte fetal ${ }^{5-8}$. El parto prematuro tiene implicaciones importantes en la morbimortalidad infantil; se ha relacionado con alto riesgo de alteraciones en el desarrollo y complicaciones clínicas que podrían incluir enfermedad pulmonar crónica, respiratoria aguda o problemas gastrointestinales 9,10 .

El parto prematuro es un problema global; cerca del I2 \% de los nacimientos en los países de ingresos bajos ocurren tempranamente, en comparación con el $9 \%$ en los países de ingresos 


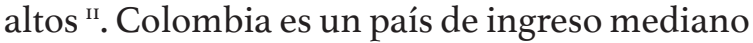
con un sistema de aseguramiento en salud con cobertura para cerca del $97 \%$ de la población ${ }^{12}$. Actualmente, en Colombia no existen publicaciones sobre los resultados materno-fetales desfavorables relacionados con la apendicectomía en mujeres embarazadas.

El objetivo primario de este estudio fue establecer factores pronóstico asociados al parto prematuro en mujeres embarazadas y el objetivo secundario fue establecer factores pronóstico para la hospitalización y la nueva hospitalización materna en la unidad de cuidados intensivos.

\section{Métodos}

\section{Fuente de datos y diseño de estudio}

Se trata de un estudio retrospectivo de cohorte llevado a cabo a partir de las bases de datos administrativos de los pacientes afiliados al sistema de salud contributivo y de las mujeres que fueron sometidas a apendicectomía durante el embarazo, entre enero de 2013 y noviembre de 20I6. La cohorte se construyó a partir de la base de datos de la Unidad de Pago por Capitación (UPC) de Colombia, proveniente del Sistema Integrado de Información de Protección Social de Colombia (SISPRO); dicha base provee información sobre los servicios de salud utilizados por la población del sistema contributivo y son reportados por las aseguradoras para su pago por parte del sistema de salud.

En dicha base de datos (UPC), se identificaron las mujeres que fueron sometidas a la cirugía dentro del periodo de estudio, por medio de los códigos de procedimiento usados en esta base y el número de la cédula de ciudadanía. Posteriormente, se cruzó con la base de datos de estadísticas vitales del Departamento Administrativo Nacional de Estadística (DANE), por medio del Registro Único de Afiliados al sistema de protección social (RUAF), donde se obtuvo el certificado de nacido vivo, el cual posee información de todo nacido vivo en Colombia. Contiene la identificación de la madre, el peso al nacer y la edad gestacional. Igualmente, se cruzó la información con el certificado de defunción del mismo siste- ma de registro mencionado anteriormente, con el fin de identificar las muertes maternas ${ }^{13}$.

\section{Población de estudio}

El estudio incluyó a todas las mujeres que fueron sometidas a apendicectomía de urgencia durante el embarazo, que pertenecían al sistema contributivo de Colombia y que tenían registro de un nacimiento vivo durante el mismo periodo del estudio. Se incluyeron I.589 mujeres en esta cohorte.

\section{Resultados}

El principal resultado estimado fue el de parto prematuro, el cual se definió como todo parto antes de 37 semanas de gestación. Además, se estimaron los resultados secundarios de mortalidad materna a los 30 días, bajo peso al nacer e ingreso, los cuales se obtuvieron de la base de estadísticas vitales por medio del registro de defunción y el de nacimiento correspondientes. La información sobre rehospitalización materna a 30 días y hospitalización en la unidad de cuidados intensivos 30 días después del procedimiento, se obtuvo de los códigos de procedimiento usados por la base de datos de la UPC.

\section{Covariables}

Las características sociodemográficas de la población en estudio incluyeron edad, lugar de atención según la región y entidad territorial. Las variables usadas como posibles factores pronóstico fueron: edad, trimestre en que se practicó la cirugía, necesidad de drenaje por peritonitis, abordaje laparoscópico, zona geográfica donde se practicó la apendicectomía e índice obstétrico de comorbilidad.

\section{Análisis estadístico}

Las características basales de la cohorte se describieron mediante frecuencias relativas para las variables categóricas, y las variables continuas se describieron por la media y su desviación estándar (DE). Los principales resultados de toda la cohorte se estimaron como tasas por 
cada Ioo cirugías. Los resultados entre presentar este resultado o no presentarlo, según edad, trimestre de embarazo, región donde se realizó la apendicectomía, abordaje quirúrgico e índice de comorbilidad obstétrica, se compararon con la prueba de ji al cuadrado ${ }^{14,15}$.

Para establecer y controlar la confusión de los factores pronóstico, se usó un modelo de regresión logística multivariado, usando la razón de momios (Odds Ratio, OR) y un intervalo de confianza del $95 \%{ }^{16}$; la significación estadística se estableció con una p menor de 0,05. Los factores incluidos en el modelo se escogieron de acuerdo con las publicaciones sobre el tema y el criterio de los investigadores. Para todos los análisis, se utilizó el software Stata $\mathrm{I}^{\mathrm{TM}}$.

\section{Resultados}

\section{Análisis descriptivo}

En total, I.589 mujeres embarazadas afiliadas al sistema contributivo de Colombia, se sometieron a apendicectomía entre 2012 y 2016 (figura I).

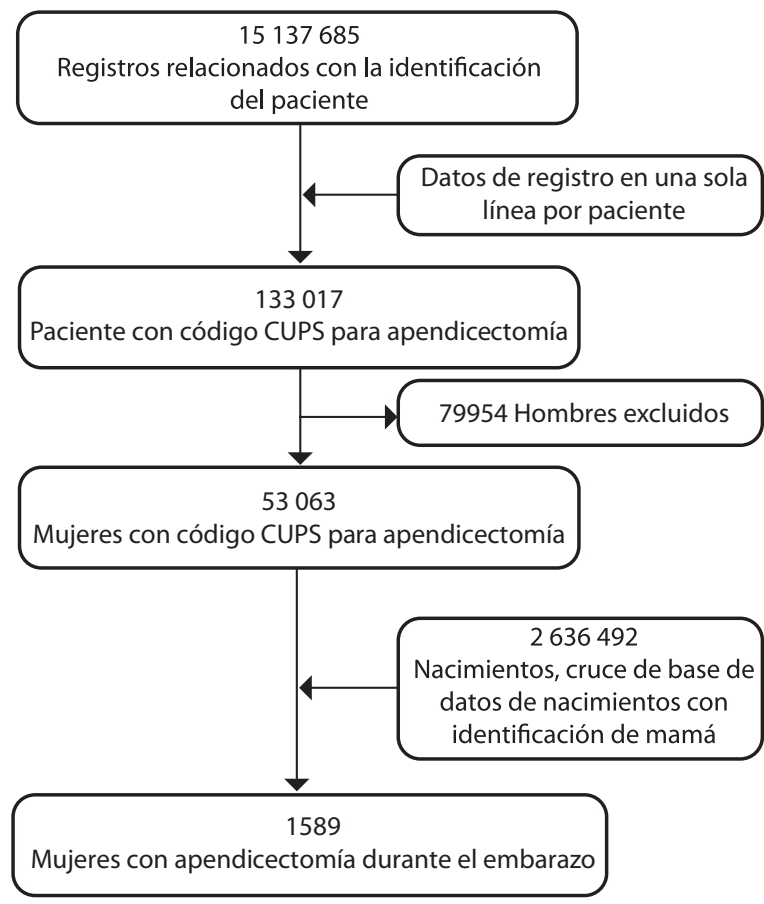

Figura 1. Flujograma para la selección de las pacientes incluidas en el estudio
Las características sociodemográficas consideradas fueron: edad, trimestre del embarazo, abordaje quirúrgico, índice obstétrico de comorbilidad y región donde se realizó la apendicectomía. La mayoría de las mujeres embarazadas estaba entre los I8 y los 35 años de edad, con una media de 26,43 \pm 5,79 años; la edad gestacional al momento del parto fue de 38 , or $\pm 2,23$ semanas; el 22,03\% requirió drenaje por peritonitis; el abordaje quirúrgico abierto fue el más frecuente; la mayoría de las mujeres gestantes se operaron durante el segundo trimestre del embarazo; el índice obstétrico de comorbilidad más frecuente $(76,63 \%)$ fue o, y Bogotá fue donde se presentó la mayor tasa de cirugías (tabla I).

Tabla 1. Características demográficas de las pacientes

\begin{tabular}{lc}
\hline Característica & $\mathbf{N}=1.589$ \\
\hline Edad (años) & $26,44 \pm 5,79$ \\
$<18$ & $74(4,66 \%)$ \\
$18-35$ & $1.336(85,97 \%)$ \\
$>35$ & $149(9,38 \%)$ \\
\hline Trimestre & \\
\hline Primero & $555(34,93 \%)$ \\
Segundo & $749(47,14 \%)$ \\
Tercero & $285(17,94 \%)$ \\
\hline Abordaje & \\
\hline Abierto & $1.492(93,9 \%)$ \\
Laparoscópico & $97(6,10 \%)$ \\
Drenaje por peritonitis & $350(22,03 \%)$ \\
\hline Índice obstétrico de comorbilidad \\
\hline 0 & $1.170(76,63 \%)$ \\
1-2 & $354(22,28 \%)$ \\
$\geq 3$ & $65(4,09 \%)$ \\
\hline Región & \\
\hline Atlántico & $264(16,61 \%)$ \\
Bogotá & $442(27,82 \%)$ \\
Otriental & $302(19,01 \%)$ \\
\hline & $372(23,41 \%)$ \\
\hline & $170(10,7 \%)$ \\
\hline
\end{tabular}


La media de peso al nacer en toda la muestra fue de 3.042 $\pm 56 \mathrm{I}, 76 \mathrm{~g}$. La más baja $(2.879 \pm 73,77 \mathrm{~g})$ se presentó en las menores de I8 años; en el grupo de 18 a 35 años fue de $3.050 \pm 15,05 \mathrm{~g}$ y, en las mayores de 35 años, de 3.058 $\pm 46,29$ g. Por otra parte, según el trimestre de embarazo en que se practicó la apendicectomía, la media de peso al nacer más baja $(2.959 \pm 35,99 \mathrm{~g})$ se encontró en el tercero; en el primero fue de $3.082 \pm 2 \mathrm{I}, 68 \mathrm{~g}$ y, en el segundo, de $3.044 \pm 2 \mathrm{I}, 08 \mathrm{~g}$. Entre las diferentes entidades territoriales, Bogotá tuvo la media más baja de peso al nacer, $2.923 \pm 29,05 \mathrm{~g}$.

\section{Análisis univariado}

Las tasas de parto prematuro, de admisión materna a unidad de cuidados intensivos, de rehospitalización materna, de bajo peso al nacer y de mortalidad materna a los 30 días, fueron de $\mathrm{I} 2,08 \%, 9,75 \%, \mathrm{I} 6,93 \%, 3,34 \%$ y $0, \mathrm{I} 3 \%$, respectivamente.

En la tabla 2 se muestran los principales resultados con respecto a las variables posiblemente predictoras. En la tabla se puede observar que el parto prematuro es más frecuente en menores de 18 años, en el tercer trimestre, con un índice de comorbilidad de 3 o más y en Bogotá.

En lo que respecta a la hospitalización en la unidad de cuidados intensivos a 30 días, fue más frecuente en menores de I8 años, en el tercer trimestre, con un índice de comorbilidad de 3 o más y en la región Atlántica. Por último, la tasa de re hospitalización a 30 días fue mayor en mayores de 35 años, en el tercer trimestre, con un índice de comorbilidad de 3 o más y en los otros depar-

Tabla 2. Principales resultados del embarazo

\begin{tabular}{|c|c|c|c|c|c|c|}
\hline Característica & $\begin{array}{c}\text { PP/Total } \\
\text { (tasa por } 100 \text { cirugías) }\end{array}$ & p & $\begin{array}{c}\text { Admisión en UCI } \\
\text { a } 30 \text { días/Total } \\
\text { (tasa por } 100 \text { cirugías) }\end{array}$ & $\mathbf{p}$ & $\begin{array}{c}\text { Readmisión } \\
\text { a } 30 \text { días/Total } \\
\text { (tasa por } 100 \text { cirugías) }\end{array}$ & $\mathbf{p}$ \\
\hline \multicolumn{7}{|l|}{ Edad (años) } \\
\hline$<18$ & $16 / 74(21,62)$ & & $10 / 74(13,51)$ & & $3 / 74(17,57)$ & \\
\hline $18-35$ & $159 / 1336(11,64)$ & & $130 / 1366(9,52)$ & & $227 / 1366(16,62)$ & \\
\hline$>35$ & $17 / 149(11,41)$ & 0,04 & $15 / 149(10,07)$ & 0,52 & $29 / 149(19,46)$ & 0,67 \\
\hline \multicolumn{7}{|l|}{ Trimestre } \\
\hline Primero & $56 / 555(10,09)$ & & $39 / 555(7,03)$ & & $99 / 555(17,84)$ & \\
\hline Segundo & $82 / 749(10,95)$ & & $72 / 749(9,61)$ & & $112 / 749(14,95)$ & \\
\hline Tercero & $54 / 285(18,95)$ & 0,00 & $44 / 285(15,44)$ & 0,00 & $58 / 285(20,35)$ & 0,09 \\
\hline Drenaje de peritonitis & $53 / 350(15,14)$ & 0,05 & $69 / 350(19,71)$ & 0,00 & $81 / 350(23,14)$ & 0,00 \\
\hline \multicolumn{7}{|c|}{ Índice obstétrico de comorbilidad } \\
\hline 0 & $135 / 1,170(11,54)$ & & $92 / 1,170(7,86)$ & & $190 / 1,170(16,24)$ & \\
\hline $1-2$ & $43 / 354(12,15)$ & & $44 / 354(12,43)$ & & $61 / 354(17,23)$ & \\
\hline$\geq 3$ & $14 / 65(21,54)$ & 0,06 & $19 / 65(29,23)$ & 0,00 & $18 / 65(27,69)$ & 0,06 \\
\hline \multicolumn{7}{|l|}{ Región } \\
\hline Atlántico & $26 / 264(9,85)$ & & $44 / 264(16,67)$ & & $60 / 264(22,73)$ & \\
\hline Bogotá & $74 / 442(16,74)$ & & $50 / 442(11,31)$ & & $100 / 442(22,62)$ & \\
\hline Central & $30 / 302(9,93)$ & & $21 / 302(6,95)$ & & $26 / 302(8,61)$ & \\
\hline Oriental & $40 / 372(10,75)$ & & $25 / 372(6,72)$ & & $55 / 372(14,78)$ & \\
\hline Pacífica & $19 / 170(11,18)$ & & $13 / 170(7,65)$ & & $18 / 170(10,59)$ & \\
\hline Otros departamentos & $3 / 39(7,69)$ & 0,02 & $2 / 39(9,75)$ & 0,00 & $10 / 39(25,64)$ & 0,00 \\
\hline Total & $192 / 1.589(12,08)$ & & $155 / 1.589(9,75)$ & & 269/1.589 (16,93) & \\
\hline
\end{tabular}

PP: parto prematuro; $\mathrm{UCl}$ : unidad de cuidados intensivos 
tamentos. La necesidad de drenaje por peritonitis mostró diferencias estadísticamente significativas para todos los resultados.

\section{Análisis multivariado}

En la tabla 3 se muestra el modelo de regresión logística multivariado para el parto prematuro; se encontró aumento del riesgo en mujeres menores de 18 años en comparación con las de I8 a 35 años. La mayor razón de momios (Odds Ratio, OR) fue estadísticamente significativa ( $\mathrm{p}$ menor de 0,05) al comparar las mujeres gestantes sometidas al procedimiento en el tercer trimestre de embarazo con aquellas en el primer trimestre, cuando hubo necesidad de drenaje peritoneal, y con un índice de comorbilidad mayor de 3 en comparación a las que

Tabla 3. Factores pronóstico para parto prematuro

\begin{tabular}{|c|c|c|c|}
\hline Característica & OR & IC $_{95 \%}$ & $\mathbf{p}$ \\
\hline \multicolumn{4}{|l|}{ Edad (años) } \\
\hline$<18$ & 1,00 & & \\
\hline $18-35$ & 0,47 & {$[0,26-0,85]$} & 0,01 \\
\hline$>35$ & 0,35 & {$[0,15-0,81]$} & 0,01 \\
\hline \multicolumn{4}{|l|}{ Trimestre } \\
\hline Primero & 1,00 & & \\
\hline Segundo & 1,13 & {$[0,78-1,62]$} & 0,53 \\
\hline Tercero & 2,05 & {$[1,35-3,13]$} & 0,00 \\
\hline \multicolumn{4}{|l|}{ Abordaje } \\
\hline Laparoscópico & 0,70 & {$[0,34-1,46]$} & 0,35 \\
\hline Drenaje por peritonitis & 1,43 & {$[1,00-2,05]$} & 0,05 \\
\hline \multicolumn{4}{|c|}{ Índice obstétrico de comorbilidad } \\
\hline 0 & 1,00 & & \\
\hline $1-2$ & 1,25 & {$[0,81-1,93]$} & 0,32 \\
\hline$\geq 3$ & 2,36 & {$[1,20-4,62]$} & 0,01 \\
\hline \multicolumn{4}{|l|}{ Entidad territorial } \\
\hline Bogotá & 1,00 & & \\
\hline Antioquia & 0,35 & {$[0,18-0,66]$} & 0,00 \\
\hline Atlántico & 0,30 & {$[0,13-0,70]$} & 0,01 \\
\hline Bolívar & 0,83 & {$[0,35-1,98]$} & 0,68 \\
\hline Boyacá & 0,49 & {$[0,17-1,43]$} & 0,19 \\
\hline Cesar & 0,25 & {$[0,08-0,72]$} & 0,01 \\
\hline Cundinamarca & 0,49 & {$[0,24-0,99]$} & 0,05 \\
\hline Meta & 0,58 & {$[0,20-1,48]$} & 0,18 \\
\hline Santander & 0,66 & {$[0,35-1,21]$} & 0,18 \\
\hline Tolima & 0,55 & {$[0,20-1,48]$} & 0,23 \\
\hline Valle del Cauca & 0,54 & {$[0,30-1,00]$} & 0,05 \\
\hline Otros & 0,64 & {$[0,40-1,01]$} & 0,06 \\
\hline
\end{tabular}

tenían un puntaje de o; además, el riesgo de parto prematuro fue menor en otras entidades territoriales de Colombia en comparación con el de Bogotá.

En la tabla 4 se encuentra el modelo de regresión logística multivariado para la hospitalización en la unidad de cuidados intensivos; se encontró un aumento del riesgo en mujeres menores de 18 años en comparación con aquellas de 18 a 35 años, con drenaje por peritonitis y con aumento del índice de comorbilidad compara-

Tabla 4. Factores pronóstico para la hospitalización materna en la unidad de cuidados intensivos a 30 días

\begin{tabular}{|c|c|c|c|}
\hline Característica & OR & IC $95 \%$ & $p$ \\
\hline \multicolumn{4}{|l|}{ Edad (años) } \\
\hline$<18$ & 1,00 & & \\
\hline $18-35$ & 0,60 & {$[0,28-1,26]$} & 0,18 \\
\hline$>35$ & 0,35 & {$[0,13-0,94]$} & 0,04 \\
\hline \multicolumn{4}{|l|}{ Trimestre } \\
\hline Primero & 1,00 & & \\
\hline Segundo & 1,31 & {$[0,85-2,01]$} & 0,22 \\
\hline Tercero & 2,07 & {$[1,26-3,40]$} & 0,00 \\
\hline \multicolumn{4}{|l|}{ Abordaje } \\
\hline Laparoscópico & 1,62 & {$[0,76-3,48]$} & 0,21 \\
\hline Drenaje por peritonitis & 3,63 & {$[2,47-5,31]$} & 0,00 \\
\hline \multicolumn{4}{|c|}{ Índice obstétrico de comorbilidad } \\
\hline \multicolumn{4}{|l|}{0} \\
\hline $1-2$ & 2,12 & {$[1,35-3,32]$} & 0,00 \\
\hline$\geq 3$ & 6,02 & {$[3,07-11,81]$} & 0,00 \\
\hline \multicolumn{4}{|l|}{ Entidad territorial } \\
\hline Bogotá & 1,00 & & \\
\hline Antioquia & 0,25 & {$[0,10-0,62]$} & 0,00 \\
\hline Atlántico & 0,98 & {$[0,51-1,88]$} & 0,96 \\
\hline Bolívar & 3,85 & {$[1,79-8,27]$} & 0,00 \\
\hline Boyacá & NA & NA & NA \\
\hline Cesar & 0,47 & {$[0,17-1,30]$} & 0,14 \\
\hline Cundinamarca & 0,38 & {$[0,15-1,00]$} & 0,05 \\
\hline Meta & 0,79 & {$[0,33-1,88]$} & 0,59 \\
\hline Santander & 0,92 & {$[0,46-1,85]$} & 0,82 \\
\hline Tolima & 1,32 & {$[0,53-3,29]$} & 0,55 \\
\hline Valle del Cauca & 0,36 & {$[0,16-0,79]$} & 0,01 \\
\hline Otros & 0,65 & {$[0,37-1,13]$} & 0,13 \\
\hline
\end{tabular}

NA: no aplica (sin hospitalizaciones en la unidad de cuidados intensivos) 
do con aquellas con puntaje de 0 . Además, nuevamente se encontraron diferencias entre otras entidades territoriales y Bogotá. En la tabla 5 se muestra el modelo para rehospitalización a 30 días; solamente se encontró asociación estadística entre el drenaje peritoneal y un índice de comorbilidad de 3 o más.

\section{Discusión}

En este estudio se evaluó una muestra representativa nacional de I.589 mujeres embarazadas a quienes se les practicó apendicectomía y se reportan los resultados de esta intervención quirúrgica en Colombia y en Latinoamérica, en este grupo poblacional.

Se han diseñado múltiples estudios con el fin de comparar los resultados entre el abordaje abierto y el laparoscópico de la apendicectomía ${ }^{\mathrm{r} 7 \mathrm{1}}$. En un metaanálisis de Prodromidou, et al., que incluyó 20 estudios de 7 países desarrollados, se concluyó que la literatura científica actual no permite establecer el abordaje ideal con base en los resultados, entre ellos, el parto prematuro ${ }^{10,19}$.

Aunque existen pocos datos poblacionales no sesgados por experiencias específicas, se reportan tasas de parto prematuro entre 8,2 y II,4 por Ioo cirugías ${ }^{6,19,20}$. En el presente estudio, la tasa estimada fue de I2,06 por Ioo cirugías. Este mayor valor probablemente se puede explicar por el contexto sociodemográfico de un país en desarrollo como Colombia. Igualmente, la mayor tasa de apendicectomías se registró en el primer y el segundo trimestres de embarazo, lo que está acorde con lo revisado en la bibliografía ${ }^{21}$.

Los datos obtenidos a partir de esta cohorte muestran una asociación entre el trimestre del embarazo en el cual se practicó la apendicectomía y el parto prematuro, información que concuerda con lo encontrado en otros estudios ${ }^{22,23,24}$. Esto puede explicarse porque, con el aumento de la edad gestacional, se pierde la correlación diagnóstica, lo cual resulta en una mayor tasa de perforación y, por consiguiente, de complicaciones que incluyen la peritonitis ${ }^{25,26}$.

Por otro lado, la datos existentes sugieren que la reacción inflamatoria puede desencadenar los
Tabla 5. Factores pronóstico para la rehospitalización a los 30 días

\begin{tabular}{|c|c|c|c|}
\hline Característica & OR & IC $_{95 \%}$ & $\mathbf{p}$ \\
\hline \multicolumn{4}{|l|}{ Edad (años) } \\
\hline$<18$ & 1,00 & & \\
\hline $18-35$ & 0,98 & {$[0,52-1,85]$} & 0,96 \\
\hline$>35$ & 0,99 & {$[0,44-2,22]$} & 0,98 \\
\hline \multicolumn{4}{|l|}{ Trimestre } \\
\hline Primero & 1,00 & & \\
\hline Segundo & 0,83 & {$[0,61-1,12]$} & 0,22 \\
\hline Tercero & 1,00 & {$[0,68-1,47]$} & 0,99 \\
\hline \multicolumn{4}{|l|}{ Abordaje } \\
\hline Laparoscópico & 1,01 & {$[0,56-1,80]$} & 0,98 \\
\hline Drenaje por peritonitis & 1,64 & {$[1,20-2,23]$} & 0,00 \\
\hline \multicolumn{4}{|c|}{ Índice obstétrico de comorbilidad } \\
\hline 0 & 1,00 & & \\
\hline $1-2$ & 1,15 & {$[0,78-1,70]$} & 0,49 \\
\hline$\geq 3$ & 2,16 & {$[1,16-4,00]$} & 0,02 \\
\hline \multicolumn{4}{|l|}{ Entidad territorial } \\
\hline Bogotá & 1,00 & & \\
\hline Antioquia & 0,18 & {$[0,09-0,38]$} & 0,00 \\
\hline Atlántico & 0,82 & {$[0,48-1,40]$} & 0,46 \\
\hline Bolívar & 0,54 & {$[0,22-1,33]$} & 0,18 \\
\hline Boyacá & 1,04 & {$[0,49-2,20]$} & 0,92 \\
\hline Cesar & 1,60 & {$[0,85-3,00]$} & 0,14 \\
\hline Cundinamarca & 0,71 & {$[0,41-1,22]$} & 0,21 \\
\hline Meta & 0,44 & {$[0,20-0,95]$} & 0,04 \\
\hline Santander & 0,41 & {$[0,22-0,77]$} & 0,01 \\
\hline Tolima & 0,91 & {$[0,42-1,99]$} & 0,81 \\
\hline Valle del Cauca & 0,33 & {$[0,17-0,61]$} & 0,00 \\
\hline Otros & 0,61 & {$[0,40-0,92]$} & 0,02 \\
\hline
\end{tabular}

mecanismos de parto, a partir de una desregulación de las vías inflamatorias, riesgo que se aumenta a medida que transcurre el embarazo ${ }^{27,28}$; por lo tanto, aunado a la presencia de peritonitis, se explica el riesgo de presentar los diferentes resultados: parto prematuro, hospitalización en la unidad de cuidados intensivos y rehospitalización materna, observados en este estudio.

En las mujeres jóvenes embarazadas, existe un riesgo acumulado de complicaciones relacionadas con la apendicitis aguda, hecho que se 
puede explicar por pertenecer al grupo etario con mayor incidencia de esta enfermedad ${ }^{29} \mathrm{y}$, por otra parte, por los riesgos inherentes al embarazo en adolescentes, entre ellos, el de mayor frecuencia de parto prematuro ${ }^{30-32}$. Por otro lado, se encontró que el resultado en el peso al nacer tiene los mismos factores que el parto prematuro, hecho que puede explicarse por la prematuridad de los recién nacidos.

En el presente estudio se incluyó el análisis del índice obstétrico de comorbilidad, el cual ha servido para predecir una lesión terminal de órgano, muerte y hospitalización en la unidad de cuidados intensivos ${ }^{33}$. En la revisión bibliográfica, no se encontró que se hubiera usado en el contexto de cirugía durante el embarazo. En este estudio, el índice obstétrico de comorbilidad fue un predictor individual para parto prematuro, hospitalización en la unidad de cuidados intensivos a 30 días y rehospitalización.

Al ser Bogotá la principal ciudad de Colombia, agrupa la mayor cantidad de pacientes de más alta complejidad, incluyendo mujeres embarazadas con alto riesgo obstétrico provenientes de la periferia del país, en donde se cuentan con servicios de salud de menor calidad y recursos limitados, lo cual puede justificar parte del riesgo calculado para esta ciudad con cada uno de los resultados; esta situación puede acarrear demoras en la atención y, con ello, impacto en la morbilidad y la mortalidad maternas y fetales.

La principal limitación de este estudio reside en el hecho de tener como fuente de información los datos retrospectivos de un registro de datos administrativos, por lo que no fue posible controlar diversas variables clínicas que pudieron haber afectado la asociación con los resultados. En casos de parto prematuro, otras posibles causas ajenas a la intervención quirúrgica podrían justificar el resultado en algunos casos ${ }^{34}$. No obstante, parece que la apendicectomía es un estímulo suficiente para desencadenar un parto prematuro ${ }^{20,21}$.

El sistema contributivo de salud de Colombia incluye una población con condiciones sociodemográficas superiores a las de los habitantes adscritos al régimen subsidiado, por lo que se podría asumir que el acceso a los servicios de salud se ve influenciado por esta situación y, por consiguiente, también los resultados más desfavorables. Aun así, la muestra de este estudio es representativa de la población colombiana y, por lo tanto, da información fiable sobre los resultados evaluados.

\section{Conclusiones}

Este estudio establece los factores pronóstico asociados con el parto prematuro en mujeres embarazadas a quienes se les practicó apendicectomía. Se encontró que la tasa de parto prematuro estuvo asociada con la edad menor de i8 años, la cirugía durante el tercer trimestre y un índice de comorbilidad de 3 o menor. Además, se encontraron diferencias regionales que sugieren una concentración de los servicios obstétricos de alta complejidad.

\section{Cumplimiento de normas éticas}

Conflictos de interés. Ninguno declarado

Consentimiento informado. Este estudio es una evaluación retrospectiva de las bases de datos del sistema de salud, y como tal, no hay necesidad de un consentimiento informado. El estudio fue aprobado por el Comité de Ética de la Facultad de Medicina de la Universidad Nacional de Colombia.

Financiación. Recursos propios de los investigadores y de la Universidad Nacional de Colombia.

\section{Referencias}

I. Barber-Millet S, Bueno-Lledó J, Granero-Castro P, Gómez-Gavara I, Ballester-Pla N, García-Domínguez R. Update on the management of non-obstetric acute abdomen in pregnant patients. Cir Esp EnglEd. 2016;94:257-65. https://doi.org/IO.IOI6/j.ciresp.2OI5.II.ooI

2. Pearl J, Price R, Richardson W, Fanelli R. Guidelines for diagnosis, treatment, and use of laparoscopy for surgical problems during pregnancy. Surg Endosc. 20II;25:3479. https://doi.org/IO.IOO7/sO0464-OII-I927-3

3. Augustin G, Majerovic M. Non-obstetrical acute abdomen during pregnancy. Eur J Obstet Gynecol Reprod Biol. 2007;13I:4-I2. https://doi.org/Io.IoI6/j.ejogrb.2006.07.052 
4. Kuy S, Roman SA, Desai R, Sosa JA. Outcomes following cholecystectomy in pregnant and nonpregnant women. Surgery. 2009;I46:358-66. https://doi. org/IO.IoI6/j.surg.2009.03.033

5. Allaert SEG, Carlier SPK, Weyne LPG, Vertommen DJ, Dutré PEI, Desmet MB. First trimester anesthesia exposure and fetal outcome. A review. Acta Anaesthesiol Belg. 2007;58:I19-23.

6. Cohen-Kerem R, Railton C, Oren D, Lishner M, Koren G. Pregnancy outcome following non-obstetric surgical intervention. Am J Surg. 2005;190:467-73. https://doi.org/IO.IoI6/j.amjsurg.2005.03.033

7. Balinskaite V, Bottle A, Sodhi V, Rivers A, Bennett $\mathrm{PR}$, Brett SJ, et al. The risk of adverse pregnancy outcomes following nonobstetric surgery during pregnancy: Estimates from a retrospective cohort study of 6.5 million pregnancies. Ann Surg. 2017;266:260-6. https://doi.org/IO.I097/SLA.00oooooooooo1976

8. Aylin P, Bennett P, Bottle A, Brett S, Sodhi V, Rivers A, et al. Estimating the risk of adverse birth outcomes in pregnant women undergoing non-obstetric surgery using routinely collected NHS data: An observational study. Southampton (UK): NIHR Journals Library; 2016. Fecha de consulta: 24 de marzo de 20I9. Disponible en: http://www.ncbi.nlm.nih.gov/books/NBK390252/

9. Behrman RE, Butler AS, editors. Committee on Understanding Premature Birth and Assuring Healthy Outcomes. Preterm Birth: Causes, Consequences, and Prevention [Internet]. Washington, D.C.: National Academies Press (US); 2007 Fecha de consulta: 24 de marzo de 2019. Disponible en: http://www.ncbi.nlm.nih.gov/ books/NBKII362/

9. Behrman RE, Butler AS, editors. Committee on Understanding Premature Birth and Assuring Healthy Outcomes. Preterm Birth: Causes, Consequences, and Prevention [Internet]. Washington, D.C.: National Academies Press (US); 2007 Fecha de consulta: 24 de marzo de 20I9. Disponible en: http://www.ncbi.nlm.nih.gov/ books/NBKII362/

Io. Saigal S, Hoult LA, Streiner DL, Stoskopf BL, Rosenbaum PL. School difficulties at adolescence in a regional cohort of children who were extremely low birth weight. Pediatrics. 2000;105:325-3I. https://doi.org/I0.I542/ peds.I05.2.325

II. Blencowe H, Cousens S, Oestergaard MZ, Chou D, Moller A-B, Narwal R, et al. National, regional, and worldwide estimates of preterm birth rates in the year 2010 with time trends since 1990 for selected countries: A systematic analysis and implications. Lancet Lond Engl. 2012;379:2162-72. https://doi.org/IO.IOI6/SoI40-6736(I2)60820-4

I2. Organización para la Cooperación y el Desarrollo Económicos (OCDE). OECD Reviews of Health Systems: Colombia, 20I5 Fecha de consulta: Junio de 2019
Disponible en: https:/www.oecd-ilibrary.org/content/ publication/9789264248908-en

13. Cendales R, Pardo C. Quality of death certification in Colombia. Colomb Médica. 20I8;49:I2I-I27. https://doi.org/I0.25I00/cm.v49ir.3155

I4. Bateman BT, Mhyre JM, Hernández-Diaz S, Huybrechts KF, Fischer MA, Creanga AA, et al. Development of a comorbidity index for use in obstetric patients. Obstet Gynecol. 2013;122: 957-65. https://doi.org/IO.I097/AOG.oboi3e3182a603bb

I5. Metcalfe A, Lix L, Johnson J, Currie G, Lyon A, Bernier $\mathrm{F}$, et al. Validation of an obstetric comorbidity index in an external population. Bjog. 2015;I22:I748-55. https://doi.org/IO.IIII/I47I-0528.I3254

I6. Peduzzi P, Concato J, Kemper E, Holford TR, Feinstein AR. A simulation study of the number of events per variable in logistic regression analysis. J Clin Epidemiol. I996;49:1373-9. https://doi.org/IO.IOI6/So8954356(96)00236-3

I7. Walsh CA, Tang T, Walsh SR. Laparoscopic versus open appendicectomy in pregnancy: A systematic review. Int J Surg Lond Engl. 2008;6:339-44. https://doi.org/IO.IoI6/j.ijsu.2008.oI.0o6

I8. Wilasrusmee C, Sukrat B, McEvoy M, Attia J, Thakkinstian A. Systematic review and meta-analysis of safety of laparoscopic versus open appendicectomy for suspected appendicitis in pregnancy. Br J Surg. 20I2;99:I470-8. https://doi.org/IO.IOO2/bjs.8889

19. Prodromidou A, Machairas N, Kostakis ID, Molmenti E, Spartalis E, Kakkos A, et al. Outcomes after open and laparoscopic appendectomy during pregnancy: A meta-analysis. Eur J Obstet Gynecol Reprod Biol. 20I8;225:40-50. https://doi.org/IO.IOI6/j. ejogrb.20I8.04.0IO

2o. Ibiebele I, Schnitzler M, Nippita T, Ford JB. Appendicectomy during pregnancy and the risk of preterm birth: A population data linkage study. Aust N Z J Obstet Gynaecol. 20I9;59:45-53. https://doi.org/IO.IIII/ajo.I2807

2I. Wei P-L, Keller JJ, Liang H-H, Lin H-C. Acute appendicitis and adverse pregnancy outcomes: A nationwide population-based study. J Gastrointest Surg. 20I2;I6:I204-II. https://doi.org/I0.I007/sII605-0I2-I858-x

22. Sadot E, Telem DA, Arora M, Butala P, Nguyen SQ, Divino CM. Laparoscopy: A safe approach to appendicitis during pregnancy. Surg Endosc. 2010;24:383-9. https://doi.org/I0.I007/s00464-009-057I-7

23. Kammerer WS. Nonobstetric surgery during pregnancy. Med Clin North Am. 1979;63:I157-64. https://doi. org/IO.IOI6/Soo25-7125(I6)31633-9

24. Yilmaz HG, Akgun Y, Bac B, Celik Y. Acute appendicitis in pregnancy - risk factors associated with principal outcomes: A case control study. Int J Surg. 2007;5:192-7. https://doi.org/IO.IOI6/j.ijsu.2006.05.005 
25. Bhandari TR, Shahi S, Acharya S. Acute appendicitis in pregnancy and the developing world. International Scholarly Research Notices. 20I7. Fecha de consulta: 24 de marzo de 2019. Disponible en: https://www.hindawi.com/journals/isrn/20I7/2636759/ https://doi.org/IO.II55/20I7/2636759

26. Davoodabadi A, Davoodabadi H, Akbari H, Janzamini M. Appendicitis in pregnancy: Presentation, management and complications. Zahedan J Res Med Sci. 20I6;18(7):e7557.. Fecha de consulta: 24 de marzo de 2019. Disponible en: http://zjrms.com/en/articles/7557.html https://doi.org/IO.I7795/zjrms-7557

27. Ravanos K, Dagklis T, Petousis S, Margioula-Siarkou C, Prapas Y, Prapas N. Factors implicated in the initiation of human parturition in term and preterm labor: A review. Gynecol Endocrinol. 2015;31:679-83. https://doi.org/Io.3I09/09513590.2015.I076783

28. Lim R, Barker G, Lappas M. Activation of AMPK in human fetal membranes alleviates infection-induced expression of pro-inflammatory and pro-labour media- tors. Placenta. 2015;36:454-62. https://doi.org/IO.IOI6/j. placenta.2015.0I.007

29. Humes DJ, Simpson J. Acute appendicitis. BMJ. 2006;333:530-4.https://doi.org/Io.II36/bmj.38940.664363. $\mathrm{AE}$

30. Lao TT, Ho LF. The obstetric implications of teenage pregnancy. Hum Reprod Oxf Engl. 1997;I2:2303-5. https://doi.org/IO.I093/humrep/I2.I0.2303

3I. Black AY, Fleming NA, Rome ES. Pregnancy in adolescents. Adolesc Med State Art Rev. 2012;23:I23-38, xi.

32. Kumar A, Singh T, Basu S, Pandey S, Bhargava V. Outcome of teenage pregnancy. Indian J Pediatr. 2007;74:927-3I. https://doi.org/IO.IO07/sI2098-007-0I7I-2

33. Bateman BT, Gagne JJ. The Obstetric Comorbidity Index predicts severe maternal morbidity. BJOG Int J Obstet Gynaecol. 2015;I22:I756-I756. https://doi. org/IO.IIII/I47I-0528.I3297

34. Frey HA, Klebanoff MA. The epidemiology, etiology, and costs of preterm birth. Semin Fetal Neonatal Med. 2016;2I:68-73. https://doi.org/IO.IOI6/j.siny.20I5.I2.oII 